\title{
Uncooled photodetectors for infrared thermography
}

\author{
by J. Pawluczyk, M. Grudzień, H. Mucha, Z. Nowak, Z. Orman, \\ M. Romanis and J. Piotrowski
}

Vigo - System Ltd., Swietlikow 3, 01-397 Warsaw, Poland

\begin{abstract}
:
An advanced photovoltaic detector is reported, based on monolithic $\mathrm{Hg}_{1-x} \mathrm{Cd}_{\mathrm{x}} \mathrm{Te}$ heterostructure with 3-D architecture. It operates in the MWIR or LWIR range at ambient temperature or under thermoelectrical cooling. Since the device operates at zero bias mode, it does not exhibit low frequency noise. The measurements show the possibility to achieve detectivity of $\approx 1 \cdot 10^{9} \mathrm{cmHz} z^{1 / 2} / \mathrm{W}$ at the 8-9 $\mu \mathrm{m}$ range. Potentially, the devices can be assembled in large focal plane arrays. This will enable obtaining a NETD of less than $0.1 \mathrm{~K}$ for staring thermal imagers operating with $f / 2$ optics and $50 / \mathrm{sec}$. frame rate.
\end{abstract}

\section{Introduction}

Infrared photodetectors are the devices of choice for high quality infrared thermography and other demanding infrared systems. When operating in the middle $(3-8 \mu \mathrm{m})$ and long $(8-14 \mu \mathrm{m})$ wavelength range, these photodetectors require cryogenic cooling to achieve useful performance. The need for cooling is a major limitation factor for widespread use of infrared technology. Affordable infrared systems, such as infrared imaging cameras, require cost-effective detectors that operate without cooling or, at least, at temperatures compatible with long-life, low power and low cost coolers.

Although for some applications thermal detector focal plane arrays have been used successfully [1], in most cases photon detectors are still the best option. In general, the performance of thermal detectors is modest, they suffer from slow response, and they are not very useful in applications requiring multispectral detection.

Recently, a number of concepts to improve performance of photodetectors operating at near room temperatures have been proposed [2][3][4][5][6][7][8]. Also, recent results show that usable performance can be achieved even for wavelengths exceeding $10 \mu \mathrm{m}$, without the need for cryogenic cooling.

In this paper we describe our recent theoretical and experimental efforts aimed at the elimination, or at least reduction, of cooling requirements of infrared photodetectors. We report an advanced photovoltaic detector operating in the MWIR and LWIR range at ambient temperature or under thermoelectrical cooling.

\section{Performance of infrared photodetectors for thermal cameras: requirements and fundamental limits}

Thermal resolution of infrared thermal systems is usually characterized by the noise equivalent temperature difference NETD [9], which is required to be not greater than $0.1 \mathrm{~K}$.

Thermal resolution improves with an increase of the detector area. On the other hand, increasing detector area results in reduced spatial resolution. Hence, a reasonable compromise between requirement of high thermal and spatial resolution has to be achieved. Improvement of thermal resolution without spatial resolution worsening may be obtained by:

a) increase of detector area combined with corresponding increase of focal length and the objective aperture,

b) improved detector performance,

c) increased number of detectors.

\footnotetext{
*Correspondence by e-mail: jpiotr@vigo.com.pl
} 
Aperture increase is undesirable because it increases size, mass and price of an IR system. It is more appropriate to use a detector with a higher detectivity. Another option is the application of multi-element sensor. This leads to reduction of each element bandwidth, proportionally to the number of elements, for the same frame rate and other parameters.

Consider the use of a photodetector that is approximated by an ideal photon counter peak performance with a certain cutoff wavelength. Detectivity of $1.9 \cdot 10^{8} \mathrm{cmHz}^{1 / 2} \mathrm{~W}$, $2.3 \cdot 10^{8} \mathrm{cmHz}^{1 / 2} \mathrm{~W}$ and $2 \cdot 10^{9} \mathrm{cmHz}^{1 / 2} \mathrm{~W}$ is necessary to obtain a $0.1 \mathrm{~K}$ NETD for $10 \mu \mathrm{m}$, $9 \mu \mathrm{m}$ and $5 \mu \mathrm{m}$ cutoff wavelength photon counter detectors, respectively, when the detector operates with $\mathrm{f} / 2$ optics and have an area of $50 \times 50 \mu \mathrm{m}$. Can this be achieved at near room temperature using photodetectors?

The Auger thermal generation-recombination limits the performance of intrinsic photodetectors operating at near room temperatures [3][4]. The detectivity of such detectors has been calculated as a function of wavelength and temperature of operation. This was performed for $10^{14} \mathrm{~cm}^{-3}$ donor doping, as it is the lowest doping achievable at present in a controllable manner. The BLIP detectivity has been determined for field of view equal $2 \pi$, $300 \mathrm{~K}$ background temperature and quantum efficiency equal to unity.

From this calculation we learn that

- liquid nitrogen cooling potentially makes possible to achieve the BLIP performance in the whole $2-20 \mu \mathrm{m}$ range,

- $200 \mathrm{~K}$ cooling, achievable with Peltier coolers, would be sufficient for BLIP operation in the middle and short wavelength regions $(<5 \mu \mathrm{m})$,

- the performance of the uncooled photodetectors is not sufficient to achieve the required $0.1 \mathrm{~K}$ thermal resolution, if the detector operates with $\mathrm{f} / 2$ optics and have an area of $50 \times 50 \mu \mathrm{m}$.

\section{Ways to improve the performance without cooling}

There are some ways to improve the performance of the photodetectors without cooling. One of them is the suppression of the Auger generation-recombination processes. A moderate $p$-type doping of the absorber region is widely used for some suppression of the Auger mechanisms [2][3][4]. More efficient suppression can be obtained with non-equilibrium depletion of the semiconductor [3]. The non-equilibrium mode devices suffer from a high level of flicker noise that makes them useless for imaging applications. Band gap engineering has been proposed to reduce Auger processes but no practical devices are available at present.

A possible way is also to reduce the physical volume of the semiconductor, thus reducing the amount of thermal generation. This must be done without decrease of quantum efficiency, optical area and field of view of the detector.

Reduction of thickness. Thickness of the active region of the detector can be significantly reduced by enhanced absorption of the radiation. This can be achieved by making use of multiple pass of radiation with a backside reflector. Even more efficient is the use of interference phenomena to set up a resonant cavity within the photodetector [2][4]. It should be noted that the interference effects strongly modify spectral response of the device. The gain due to the optical cavity can be achieved only in narrow spectral regions. This may be an important limitation for some applications that require wide spectral band. In practice, infrared systems usually operate in a spectral band (e.g. atmospheric windows) and the use of resonant cavities may yield significant gains. Another limitation comes from the fact that efficient optical resonance occurs only for near perpendicular incidence and is less effective for oblique incidence. This limits the use of the devices with fast optics.

Reduction of physical area of the detector. A possible way to improve the performance of infrared photodetector is to increase the apparent "optical" size of the detector in comparison with the actual physical size using a suitable concentrator that compresses impinging infrared radiation. This must be achieved without reduction of acceptance angle, or 
at least, with limited reduction of angles as required for fast optics of infrared systems. An efficient possible way to achieve an effective concentration of radiation is to immerse the photodetector to the hemispherical or hyperhemispherical lens [2][4][10][11][12]

Several factors must be taken into account in practical realization of the optically immersed detectors. The practical use of the immersion technology has been limited due to problems in detector mechanical matching with the lens material, as well as severe transmission and reflection losses. Another limitation is due to the limited acceptance angle of the devices as a result of the total reflection at the lens-glue interface.

The problems of matching of detectors with immersion lenses have been solved by using monolithic technology developed at VIGO [4]. This technology is based on epitaxy of $\mathrm{HgCdZnTe}$ on (CdZn)Te. The HgCdZnTe serves here as the sensitive element, while the immersion lens is formed directly in the transparent $(\mathrm{CdZn}) \mathrm{Te}$ substrate. Monolithic optical immersion results in significant improvement of detector parameters.

The gain factors achieved with the hyperhemispherical immersion in (CdZn)Te are substantially higher compared to those for the hemispherical one. The immersion to an aplanatic hyperhemispherical lens results in the increase of the apparent linear detector size by the factor $n^{2}$, where $n$ is the refraction index of lens material, while in the hemispherical one - only by the factor of $\mathrm{n}$. The hyperhemispherical immersion may restrict the acceptance angle of the detector and requires more stringent manufacturing tolerances. These restrictions depend on the refraction coefficient of the lens. For $(\mathrm{Cd}, \mathrm{Zn}) \mathrm{Te}$, however, they are not so severe as for germanium lenses and have no practical importance in many cases. For example, $\mathrm{f} / \#$ of the main optical system is limited to about 1.3 by the CdZnTe immersion lens ${ }^{*}$.

\section{Practical devices with improved performance}

The methods of reducing the physical volume of the detector have been widely applied in uncooled IR photodetectors of various types developed and manufactured at VIGO SYSTEM [2][4]. These devices are based on variable gap $\mathrm{Hg}_{1-x} \mathrm{Cd}_{x} \mathrm{Te}$ heterostructures grown by Isothermal Vapor Growth Epitaxy (ISOVPE) in a proprietary reusable growth system, which also enables in situ doping with foreign impurities [13].

As an example, an advanced photovoltaic device with multiple heterojunctions is shown in Fig. 1. The linear trenches in the epilayer were chemically etched using Br/ethylene glycol or $\mathrm{Br} / \mathrm{HBr}$ solutions. Etching was followed by shallow ion beam milling using a Kaufman-type ion gun. The sample was placed at $\approx 45^{\circ}$ angle to the direction of the $\mathrm{Ar}^{+}$beam so that only one wall of the trench was exposed to the beam. The ion beam milling results in $n$-type conversion and formation of $\mathrm{n}^{+} \mathrm{p}$ junctions on the uppermost surface and on one side of the trench walls. Due to the grading of the epilayer composition and the dopants concentration varying with depth, the final structure resembles an $3-D n^{+} \pi p p$ diode. The metalization was deposited to provide external contact to the device and to short-circuit the $n^{+} p$ junctions, effectively connecting side-by-side diodes in series. For an epilayer $\sim 8 \mu \mathrm{m}$ thick, the minimum practical period of the structure using existing wet etching technology is $\approx 10 \mu \mathrm{m}$.

Fig. 2 shows the performance versus wavelength of $\mathrm{HgCdTe}$ photovoltaic devices manufactured using the multi-heterojunction technology and monolithic optical immersion in the CdZnTe lens [7][8]. The devices were based on multiple junctions structure with a period of $15-30 \mu \mathrm{m}$. Generally, uncooled $10.6 \mu \mathrm{m}$ photovoltaic devices operating at room temperature, have better performance than that of photoelectromagnetic devices operating under the same conditions. Peltier cooled devices exhibit performance that is comparable to photoconductors operating at the same wavelength and temperature. However, in contrast to photoconductors, the multi-heterojunction photovoltaic detectors can be successfully used

\footnotetext{
${ }^{*}$ CdTe refraction index $\approx 2.7$.
} 
both at very low and very high frequencies. Heterodyne experiments indicate that the response time of LWIR devices at a wavelength of $10.6 \mu \mathrm{m}$ is only about $1 \mathrm{~ns}$.

The devices are manufactured as single element ones, optimized for any wavelength within 2-13 $\mu \mathrm{m}$ range, with active area sizes from a few micrometers to a few millimeters. Linear arrays up to 120 elements and small 2-D arrays are manufactured as custom devices.

Optical immersion has been used almost exclusively for single element devices. Applying a single immersion lens to a large array is problematic in view of optical aberrations and large lens size.

Recent efforts at Vigo are aimed at practical implementation of a concept of improved uncooled LWIR and MWIR devices proposed by Piotrowski [14]. The device is a 2-D array of small size infrared detectors, monolithically integrated with microlenses. The individual elements can be accessed individually or connected in series.

Fig. 3 shows schematically the design of the individual active element of the device. Two different configurations are used - mesa and planar structures. The device is based on monolithic $\mathrm{Hg}_{1-x} \mathrm{Cd}_{x} \mathrm{Te}$ heterostructure with $3-\mathrm{D}$ architecture, which consists of several regions:

- Absorber of infrared radiation buried in wide gap material. This is a narrow gap p-type $\mathrm{Hg}_{1-\mathrm{x}} \mathrm{Cd}_{\mathrm{x}} \mathrm{Te}$ that has been optimized for good absorption of infrared radiation and low thermal generation. A small size of the active element $(\approx 7 \times 7 \mu \mathrm{m})$ is beneficial for good collection of photogenerated charge carriers. This is especially important for uncooled LWIR devices that are based on semiconductors with a short diffusion length.

- Graded gap $\mathrm{Hg}_{1-x} \mathrm{Cd}_{\mathrm{x}} \mathrm{Te}$ layer surrounding the absorber region with gradually increasing band gap. This prevents thermal generation and recombination at surfaces of the absorber.

- Heterojunction contacts collecting photogenerated carriers. They are obtained by selective $\mathrm{p}$ and $\mathrm{n}^{+}$doping of some parts of the graded gap $\mathrm{Hg}_{1-\mathrm{x}} \mathrm{Cd}_{\mathrm{x}} \mathrm{Te}$ layer surrounding the absorber region.

- Immersion microlens formed directly in the CdZnTe substrate of the $\mathrm{Hg}_{1-\mathrm{x}} \mathrm{Cd}_{\mathrm{x}} \mathrm{Te}$ epilayer (Fig. 4). The lens concentrates incoming radiation in the absorber region. Since this region is located at the aplanatic position of the hyperhemisphere microlens, the apparent linear size of the active area is $n^{2}$ times the physical size of the absorber, where $n$ is the refraction index of the lens $(\mathrm{n} \approx 2.7$ for CdTe). Typically, the apparent optical area is a $50 \times 50 \mu \mathrm{m}$ square, which corresponds to a $7 \times 7 \mu \mathrm{m}$ size of the absorber region. Due to the small sag of the lens, matrix of the lenses can be prepared in a collective way (Fig. 4). The sag height is controlled by the time of the consecutive wet etch and ion milling.

- The heterostructure is supplied with a ZnS passivation layer and contact metalization to $\mathrm{n}^{+}$and $\mathrm{p}$-regions. These two layers also play a role of a mirror that improves quantum efficiency for weakly absorbed infrared radiation. In addition, the mirror eliminates backside incidence of thermal radiation, preventing unwanted generation of dark current and related noise [15].

Test structures of the devices have been prepared using a combination of several specific methods:

- Substrate profiling with wet chemical etching before epitaxy.

- Modified isothermal vapor phase epitaxy of $\mathrm{Hg}_{1-x} \mathrm{Cd}_{x} T e$. Selective epitaxy on profiled CdZnTe substrates is used for planar devices.

- Negative epitaxy of $\mathrm{Hg}_{1-x} \mathrm{Cd}_{\mathrm{x}}$ Te that is applied to widen the band gap of surface regions.

- Doping in situ during epitaxy and selective post growth diffusion doping.

- Multiple wet chemical etching and ion milling used for formation of microlenses.

- Vacuum deposition of dielectric and metal layers.

- Indium bump bonding. 
The devices are especially promising as uncooled 7.8 - $9.5 \mu \mathrm{m}$ detectors that can be used for thermal imagers. Initial results are encouraging, indicating the potential for achieving $\approx 10^{9} \mathrm{cmHz}^{1 / 2} \mathrm{~W}$ at $\lambda \approx 9 \mu \mathrm{m}$. This would enable thermal resolution better than $0.1 \mathrm{~K}$ for staring thermal imagers operating with $\mathrm{f} / 2$ optics. A similar design has also been used for 8-12 $\mu \mathrm{m}$ detectors for conventional and Fourier spectroscopy and for $10.6 \mu \mathrm{m} \mathrm{CO}$ laser receivers.

The device exhibits very fast response with a time constant of $\approx 1$ nanosecond. This is due to the short time required for photogenerated carriers in the small size active region to reach heavily doped contacts and a short RC time constant as well.

The advantages of the device such as convenient uncooled operation without electric or magnetic bias, easy testing, readiness to use at any moment, fast response and, last but not least, low production costs make possible large scale applications.

\section{References}

[1] "Uncooled Imaging Arrays and Systems", in Semiconductor and Semimetals, 47, pp. 116, edited by P. W. Kruse and D. D. Skatrud, 1997.

[2] J. Piotrowski, W. Galus and M. Grudzien, "Near room-temperature IR photodetectors," Infrared Phys. 31, pp. 1-48, 1990.

[3] C. T. Elliott and N. T. Gordon, "Infrared Detectors", in Handbook on Semiconductors, 4, pp. 841-936, edited by C. Hilsum, North-Holland, Amsterdam, 1993.

[4] J. Piotrowski. " $\mathrm{Hg}_{1-\mathrm{x}} \mathrm{Cd}_{\mathrm{x}} \mathrm{Te}$ Detectors," in Infrared Photon Detectors, pp. 391-494, edited by A. Rogalski, SPIE Optical Engineering Press, Bellingham, 1995.

[5] J. Piotrowski and M. Razeghi, "Improved performance of IR photodetectors with 3D gap engineering", Proc. SPIE, 2397, pp. 180-192, 1995.

[6] J. Piotrowski and W. Gawron. "Ultimate Performance of Infrared Photodetectors and Figure of Merit of Detector Material", Infrared Physics and Technology, 38, pp. 63-68, 1997.

[7] B. Musca, J. Antoszewski, J. Dell, L. Faraone J. Piotrowski, Z. Nowak, "Multi-junction $\mathrm{HgCdTe}$ long wavelength infrared photovoltaic detector for operation at near room temperature," J. Electronic Mat. 27, pp. 740 - 746, 1998.

[8] J. Piotrowski, Z. Nowak, J. Antoszewski, C. Musca, J. Dell, and L. Faraone, "A novel multi-heterojunction $\mathrm{HgCdTe}$ long-wavelength infrared photovoltaic detector for operation under reduced cooling conditions," Semicond. Sci. Technol. 13, pp. 1209-1214, 1998.

[9] E. L. Dereniak and G. D. Boreman, Infrared Detectors and Systems, J. Wiley and Sons, N.Y. 1996.

[10] N. T. Gordon, "Design of $\mathrm{Hg}_{1-x} \mathrm{Cd}_{\mathrm{x}} \mathrm{Te}$ infrared detector arrays using optical immersion with microlenses to achieve a higher operation temperature," Semicond. Sci. Technol. 8, pp. 106-109, 1991.

[11] C. L. Jones, B. E. Metthews, D. R. Purdy and N. E. Metcalfe, "Fabrication and assessment of optically immersed CdHgTe detector arrays," Semicond. Sci. Technol. 6, pp. 110-113, 1991.

[12] M. E. Motamedi, W. E. Tennant, H. O. Sankur, R. Melendes, N. S. Gluck, S. Park, J. M. Arias, J. Bajaj, J. G. Pasko, W. V. McLevige, M. Zandian, R. L. Hall, P. D. Richardson "Micro-optic integration with focal plane arrays". Optical Engineering, 36(5), 1374-1381. (May 1997).

[13] J. Piotrowski, Z. Nowak, M. Grudzień, W. Galus, K. Adamiec, Z. Djurić, V. Jović, Z. Djinović, „High capability, quasi-closed growth system for isothermal vapour phase epitaxy of (Hg,Cd)Te," Thin Solid Films, 161, pp. 157-169, 1988.

[14] J. Piotrowski, unpublished, 1999.

[15] N. T. Gordon, R. S. Hall, "MCT infrared detectors with close to radiatively limited performance at $240 \mathrm{~K}$ in the 3-5 $\mu \mathrm{m}$ band," Proc. SPIE 1999 


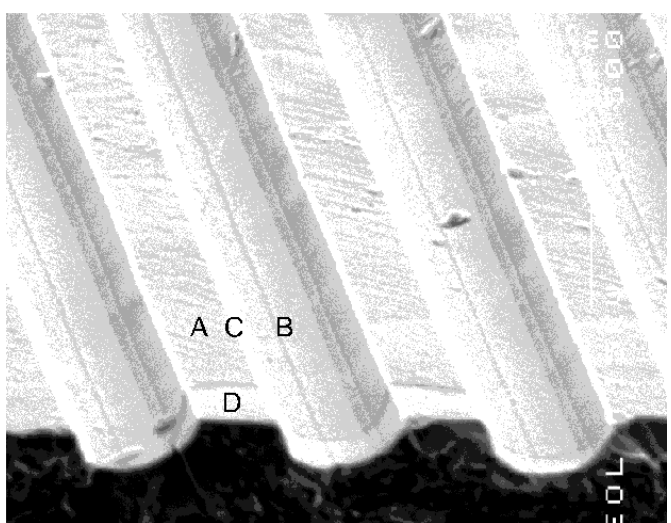

Fig. 1. SEM image of the multi-heterojunction photodetector. A-mesa structure, B- trenches, C- non-metalized wall, D- non-metalized region of the device.

a)

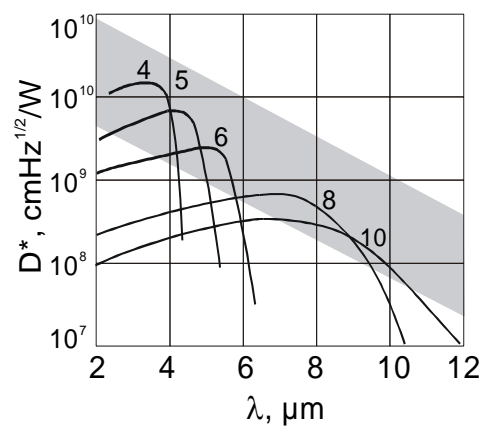

b)

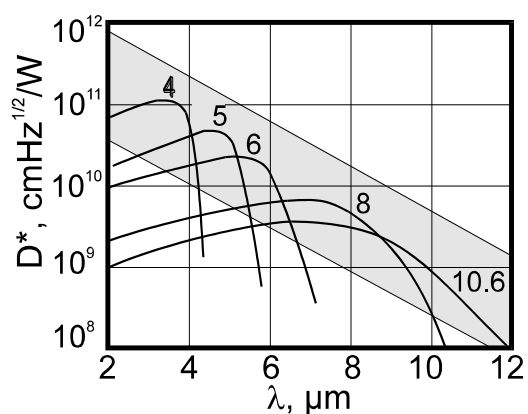

Fig. 2. Measured detectivity of multi-heterojunction photovoltaic detectors: a)- uncooled and b)- cooled with two-stage Peltier cooler. Optical immersion has been used to improve the performance.

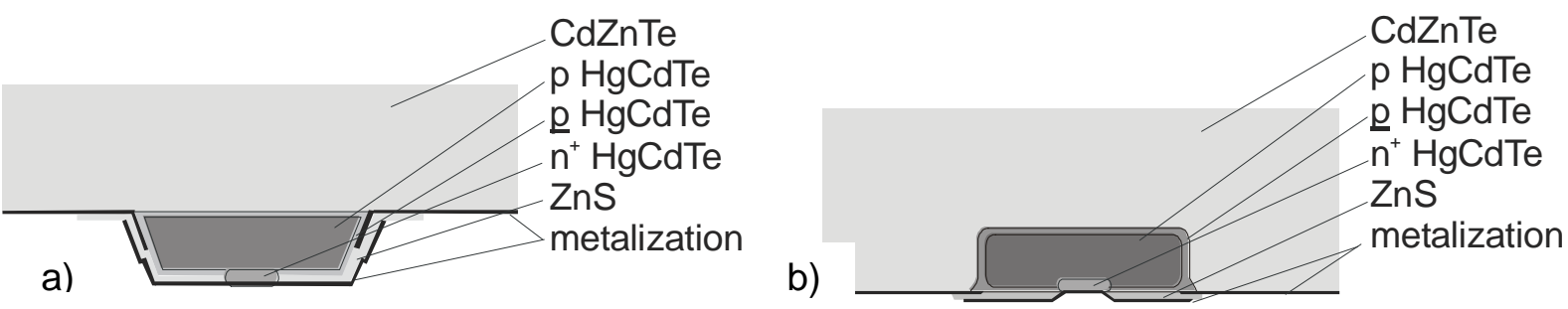

Fig. 3. Schematic cross-sections (not in scale) of two types of active elements: a) mesa type, b) planar device.
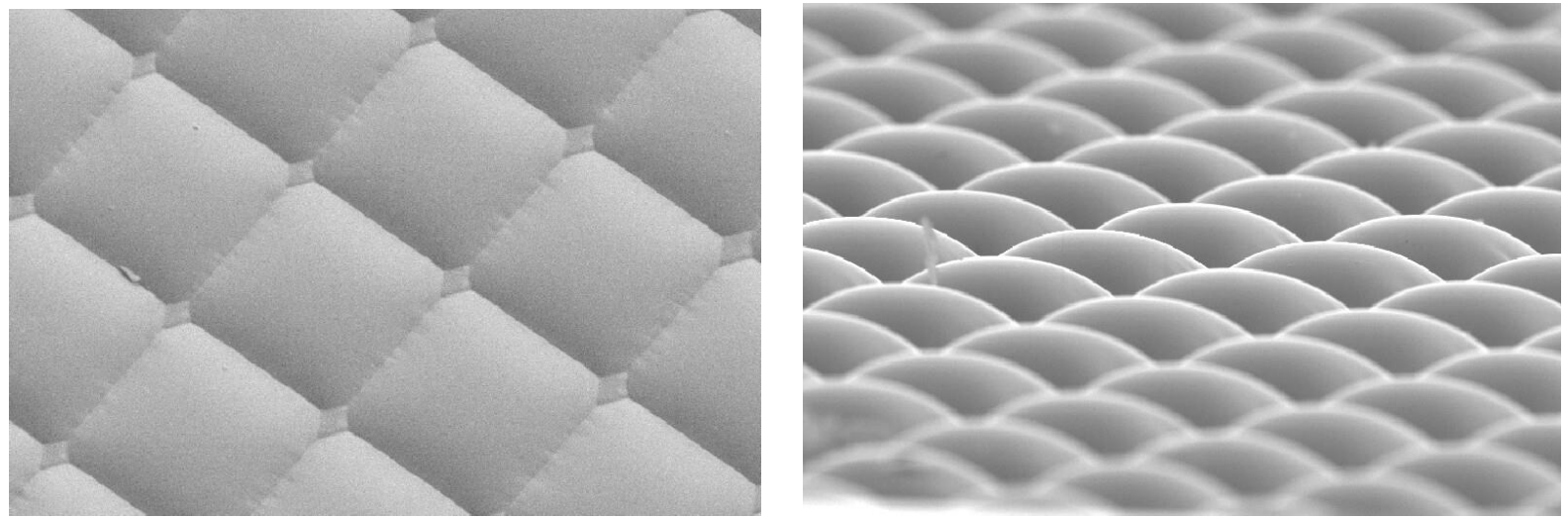

Fig. 4. SEM images of the microlens array: a) top and b) tilted side view. 